\title{
The dynamics of the two mutually coupled autonomous Van der Pol Oscillator
}

\author{
S. Sabarathinam and K. Thamilmaran \\ Centre for Nonlinear Dynamics, School of Physics, Bharathidasan University, Tiruchirappalli - 620024.
}

In this work, the dynamical behavior of the two mutually coupled Autonomous Van der Pol oscillator studied via numerical and experimental observations. We show, when the appropriate coupling co-efficient, the transition from phase synchronization to anticipatory synchronization.

The transition confirmed through similarity function. The rich nonlinear dynamical phenomena of chaos identified and confirmed through bifurcation and Lyapunov exponent spectrum analysis. The well known period doubling route to chaos are identified. The above mentioned dynamical behavior are observed in the real time hardware experimental circuit simulations.

\section{Introduction}

In nonlinear dynamics, chaos generally represent as the present does not approximately determine the future. In the last two three decades, a tremendous amount of work have been done to identifying chaos, controlling, and synchronization, etc,. Chaotic behavior exists in many natural systems, such as weather and climate. This behavior can be studied through analysis of a chaotic mathematical model, or through analytical techniques such as Lyapunov exponent spectrum analysis, power spectrum, recurrence plots and Poincar'e maps. Chaos theory has applications in several disciplines, including meteorology, sociology, physics, engineering, economics, biology, and philosophy. The Van der Pol oscillator is a classical example of self oscillatory system is now considered as very useful mathematical model that can be used in much more complicated and modified systems [9]. The Van der Pol oscillators with non linear damping which can be treated as an equivalent of mechanical self excited systems. In the autonomous flat form a single oscillator, we did not get any chaotic motion because of its dimension. But the coupled system configuration we expect the chaotic motion. In the present study, we examine the existence of the chaotic attractors both numerical and experimental investigation for two mutually coupled Van der Pol oscillator.

Studies of synchronization in oscillatory networks have been extensively reported in the various field such as physical [1], biological [2] and electrical systems [3]. Complete synchronization of chaotic oscillator has been described theoretically and observed experimentally[5]. Intention of coupled self oscillatory systems leads to new phenomena such as synchronization, hysteresis, phase Multistability, etc, [6-8]. Through the literature number of new idea's have appeared and new types of synchronization have been identified [10-13]. The chaotic systems are synchronized such they are under control of mechanisms. Such as drive-response or activepassive of the system, direct bi-directional (mutual) coupling or unidirectional (master-slave) diffusive coupling between the oscillators[14-16]. In this connection we introduce a linear feedback coupling [17] of the individual system and coupled mutually of the two systems. For a particular choice of parameter, we confirm the existence of the anticipatory synchronization via phase synchronization the coupling co-efficient is varied.

This paper organized as follows, section 2, the proposed model of the two mutually coupled autonomous Van der Pol oscillator (2AVdP) is presented. Section 3, numerical and experimental studies of the existence of the chaotic attractor in $2 \mathrm{AVdP}$, Section 4 deals with the anticipatory synchronization in a $2 \mathrm{AVdp}$ finally section 5 , we conclude our results.

\section{The Model}

Van der pol proposed a nonlinear differential equations

$x^{\prime \prime}+\mu(x 2-1) x^{*}+x=0$

The above equation is known as autonomous Van der pol oscillator (AVdp). Here, $\mu$ is the nonlinear damping. Studying the case $\mu>>1$, Van der pol discovered the importance of what has become known as relaxation oscillation [18]. The relaxation oscillation have become corner stone of geometric singular perturbation theory and play a significant role in the analysis.

The two mutually coupled autonomous Van der Pol oscillator normalized circuit equation as follows, 
$\mathrm{x}^{* 1} 1+\mu 1\left(\mathrm{x} 2^{1}-1\right) \mathrm{x}_{1}{ }+\mathrm{x}_{1}+\mathrm{Q}\left(\mathrm{x}_{2}-\mathrm{x}_{1}\right)=0$

$x^{\prime \prime} 2+\mu 2\left(x 2^{2}-1\right) x^{*}+x_{2}+Q\left(x_{1}-x_{2}\right)=0$

where $\mu 1-2$ is the nonlinear damping and $Q$ is the coupling co-efficient. For our numerical study, we split the Eq. (2) in two four first order differential equations as follows,

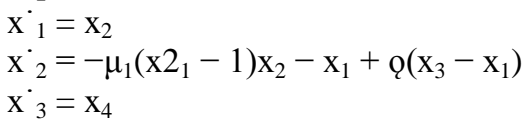

$\mathrm{x}_{4}=-\mu_{2}\left(\mathrm{x} 2_{3}-1\right) \mathrm{x}_{4}-\mathrm{x}_{3}+\mathrm{Q}\left(\mathrm{x}_{1}-\mathrm{x}_{3}\right)$

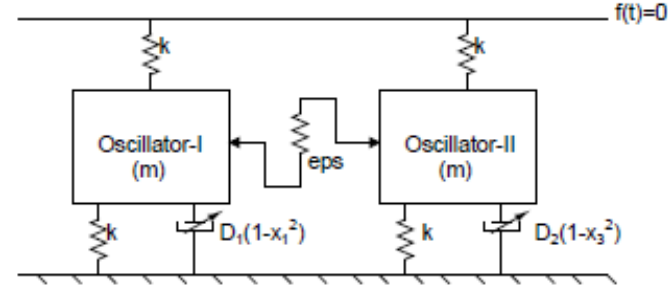

FIG. 1. Mechanical model of two mutually coupled Van der Pol oscillator. Here 'eps' represent the coupling between the two oscillator system.

In Fig. (1), shows the mechanical model of the two mutually coupled Van der Pol oscillator. K is the restoring force and the 'eps' denotes the coupling co-efficient, $\mathrm{D}_{1}\left(1-\mathrm{x}^{2}{ }_{1}\right), \mathrm{D} 2\left(1-\mathrm{x}^{2}{ }_{3}\right)$ is the nonlinear damping of the $2 \mathrm{AVdP}$. ' $\mathrm{m}$ ' is the mass of the system [9].

\section{Anticipatory Synchronization}

\section{Numerical Analysis}

Synchronization constitutes one of the most studied nonlinear phenomenon. The analysis of mutual synchronization of two weakly coupled nonlinear oscillator treated by Mayor and Gaponov [19-20]. Synchronization understood as an adjustment of rhythms of oscillating objects due to their interaction even if it is weak [21]. Van der pol oscillator constitutes the paradigmatic model to study synchronization. Quit recently, it has been discovered that two coupled non identical Van der pol oscillator can exhibit the phenomenon of anticipatory synchronization [22-26] in which the dynamical variable $\mathrm{x} 3(\mathrm{t})$ anticipate $\mathrm{x} 1(\mathrm{t})$ specifically given two slightly different oscillator in the Eq. (3). If there is a coupling between them with a coupling strength o, then one expect $\mathrm{x} 1(\mathrm{t})$ synchronize $\mathrm{x} 3(\mathrm{t}+\tau)$ in a range of value $\mathrm{Q}$, where the delay is $\tau=0$ is the time anticipate which depends on both $Q$ and the parameter characterizing the difference between the oscillators [25]. We cannot observe when the two system are identical (same parameter value). If the two system have the same parameter value, we get a complete synchronization. So, we have some mismatches needed of the two oscillators for observing anticipatory synchronization. Here we took of our proposed systems the nonlinear damping values are $\mu 1=4.0, \mu 2=4.1$ and coupling co efficient are $Q=0.11$, we observe the anticipatory synchronization shown in the Fig. (2)a. Increasing o often leads to a transition of complete synchronization at $\mathrm{Q}=1.6$. (i.e), $(\mathrm{x} 3(\mathrm{t})-\mathrm{x} 1(\mathrm{t}))=0$ as shown in the Fig. (2)b.

We have also performed a experimental study using electronic circuits that replicates to detect the anticipatory synchronization. The proposed circuits are shown in the section (IV) and we normalized the nonlinear damping values and the coupling co-efficient which are given in the 

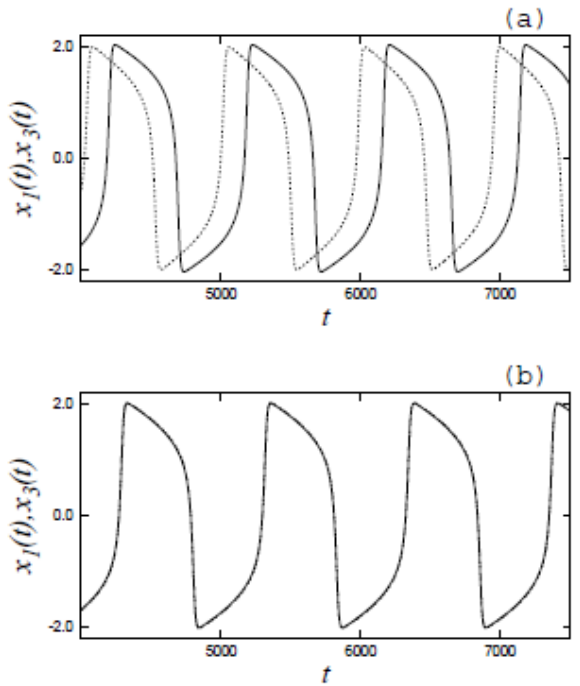

FIG. 2. Numerically observed time trajectories $(t-x 3(t), x 1(t))$ planes of (a) anticipatory synchronization and (b) complete synchronization for the particular range of cou- pling co efficient.

Same section. Figure (3) shows that the experimental observation of anticipatory synchronization (Fig. (3)a) and complete synchronization (Fig. (3)b) for the appropriate experimental component values which is fixed to matches with its numerical parameters. Following time series of drive and response system variables $\mathrm{x} 1(\mathrm{t}), \mathrm{x} 3(\mathrm{t})$ is shown in the Fig. 2(a,b) and the corresponding experimental plots are also shown in the fig. 3(a,b). From these Fig. 3(a,b), it is clearly seen that both the phase and amplitudes of the drive and response systems are coinciding, providing that the $2 \mathrm{AVdp}$ exhibits complete synchronization.

For $\mathrm{Q}=0.11$, the coupled oscillators exhibits anticipatory synchronization. Figure 2(a) and Fig. 3(a) depicts the time series plot of $x 1(t)$ and $x 3(t)$ numerically and experimentally as $v 1(\tau)$ and $v 2(\tau)$. From these figure we can observe that $\mathrm{x} 3(\mathrm{t})$ anticipates $\mathrm{x} 1(\mathrm{t})$. In other words, the response system anticipates the drive system, thereby we can infer the anticipatory synchronization of the system shown in the Eq. (3). To quantify anticipatory synchronization, we use the following similarity function [27] defined with respect to one dynamical variable, say x1 of the Van der pol oscillator,

$$
S^{2}(\tau)=\frac{\left[\left\langle x_{3}(t+\tau)\right\rangle-\left\langle x_{1}(t)\right\rangle\right]^{2}}{\sqrt{\left\langle\left(x_{3}(t)\right\rangle^{2}\left\langle x_{1}(t)\right\rangle^{2}\right)}}
$$

Where $\tau$ is the anticipation time. Anticipating synchronization between the two oscillators is characterized by the conditions Smin and $\tau 6=0$. While complete

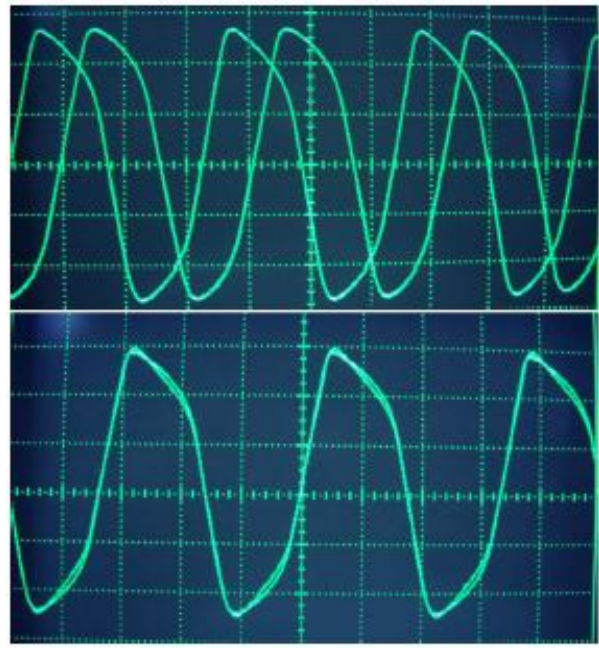

FIG. 3. Experimental observation of Anticipatory synchro- nization and complete synchronization when the coupling co-efficient are varied. The circuit components are fixed as per Section IV. 


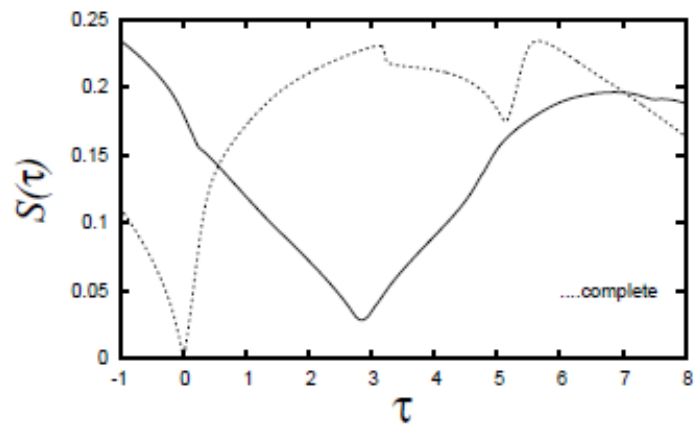

FIG. 4. Numerical calculation of similarity function for antic-ipatory and complete synchronization. Here the dashed line indicates the complete synchronization and the smooth lineindicates the anticipatory synchronization of the system.

Synchronization $\mathrm{S}(\tau)=0, \tau=0$. Numerically as the coupling strength is increased, one observes the transition from anticipatory synchronization to complete synchronization.

In the above similarity function figure express that the anticipatory synchronization and the complete synchronization of $2 \mathrm{AVdp}$. Figure (4) the dotted lines shows that complete synchronization for $\mathrm{S}(\tau)=0$ and $\tau=0$, and block line shows that anticipatory synchronization $S(\tau)=0.02926, \tau 6=0$.

\section{Quasiperiodicity in a bifurcation diagram}

In the above part discussed about that the anticipatory synchronization when the two oscillator having some mismatching parameters. Here, we analyze to changingthe mismatching parameters and to observe what kind of phenomenon can be expressed from the Eq. (3). If the mismatching is increased $\mu 1<\mu 2$. The periodicity can be broken and the quasiperiodicity are born.

The quasiperiodicity can be observed in phase portraits and power spectrum and bifurcation diagram analysis. The quasiperiodicity can be confirmed by Lyapunov spectrum analysis by $\lambda=0$. Figure (6) shows the one parameter bifurcation and its corresponding Lyapunov exponent spectrum of the coupling co-efficient in the range of $a \in(0.85,1.02)$. In this figure, the quasiperiodic exist up to $=0.90$. The two Lyapunov exponent are zero which tells that the existence of the quasiperiodic nature.
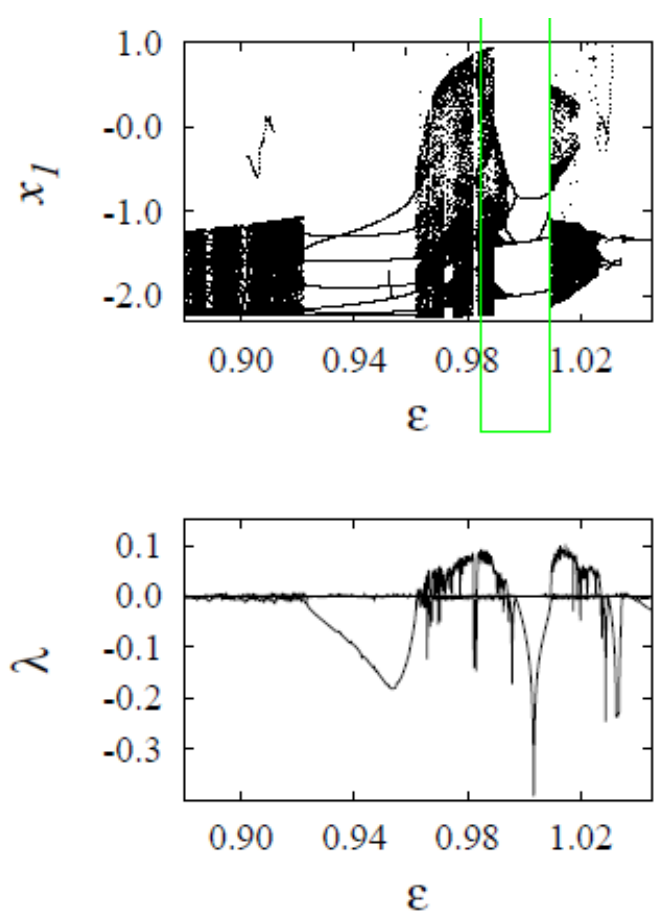

FIG. 5. numerically computed (a) Bifurcation diagram, (b) Lyapunov spectrum for the coupling coefficient in the range of $Q 2(0.85,1.02)$. 


\section{Period doubling cascades}

In the above section describes the existence of the quasiperiodic motion in a $2 \mathrm{AVdp}$. Here, we have to changing the mismatching value (i.e), $\mu 1=2.5, \mu 2=5.5$ the invariant torus is destroyed. The chaotic attractor are replacing the quasiperiodic motion when the control parameter are increased it is shown in the Fig. (6). Figure 6(a) is the full range of bifurcation diagram express the torus break through to chaoticity. In the Fig. (7) the region of the bifurcation diagram indicated the colour box in the Fig. (6). The extended region of bifurcation diagram clearly indicates the period doubling cascades and corresponding lyaponuv spectrum in Fig. 7(b) also confirm the same. The period doubling cascades are considered as a possible route to chaos. In order to see more evidently we choose some specific parameter $\mu 1,2$ and $\mathrm{o}$ and we plot again the bifurcation diagram with respect to the existing o. But, this time in a very small interval using greater step size is shown in the Fig. 7(a,b).
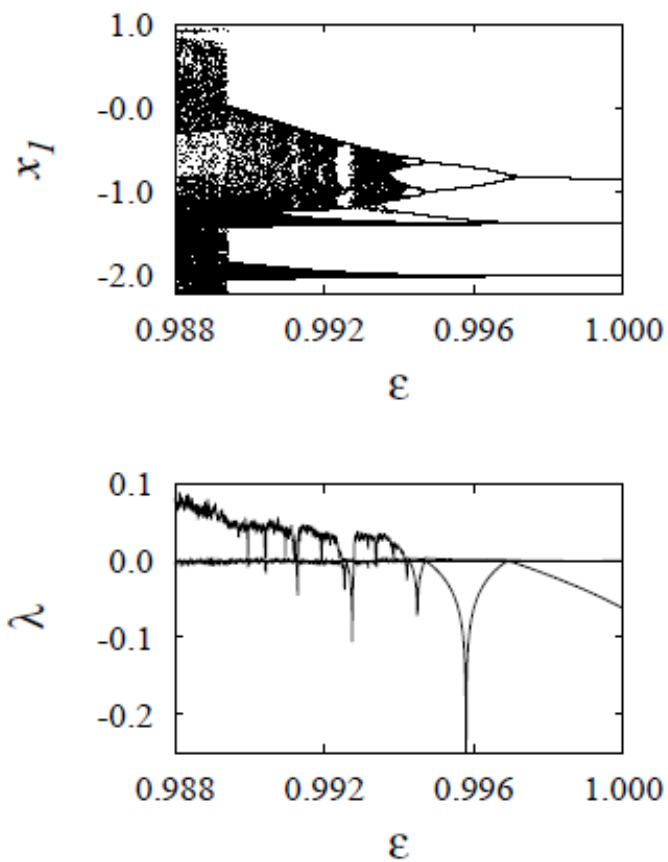

FIG. 6. Extended region of (a) Bifurcation diagram, (b) Lyaponuv spectrum in a green box of fig. 6(a).

We have also demonstrate the period doubling cascades by plotting the phase portraits shown in the Fig. (8). To do this, we keep fixed the parameter $\mu 1, \mu 2$ and we have slightly change the coupling co-efficient $Q$. In the phase portraits it is obvious that system through periodic and non-periodic region, as $Q$ increases. The value of the period doubling cascades are tabulated as follows.

\begin{tabular}{|l|l|}
\hline Fig. 7 & Numerical $\epsilon$ values \\
\hline a & 1.000 \\
b & 0.997 \\
c & 0.994 \\
d & 0.993 \\
e & 0.9927 \\
f & 0.992 \\
\hline
\end{tabular}

\section{Experimental Study}

Dynamical system (3) can be implemented as an electronic circuit shown in the fig.(9). Fig. 9(a) present the 

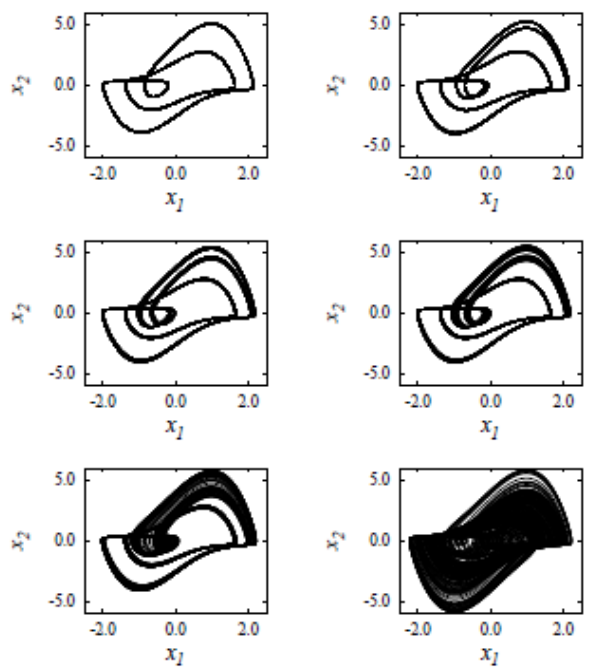

FIG. 7. Phase portraits values of $Q$, showing period doubling cascades. The values are tabulated in Table-I.

Circuit diagram and fig. 9 (b) is the laboratory realization circuit. Each indivitual oscillator shown in the red frame and is built using two capacitors, five resistors and two multipliers AD633 which introduces nonlinearity. The coupling is introduced by the resistors R7,R10,R11,R12 and the parameter R7 which is the controlling device.

Applying kirchoff's laws through A,B,C,D and we can

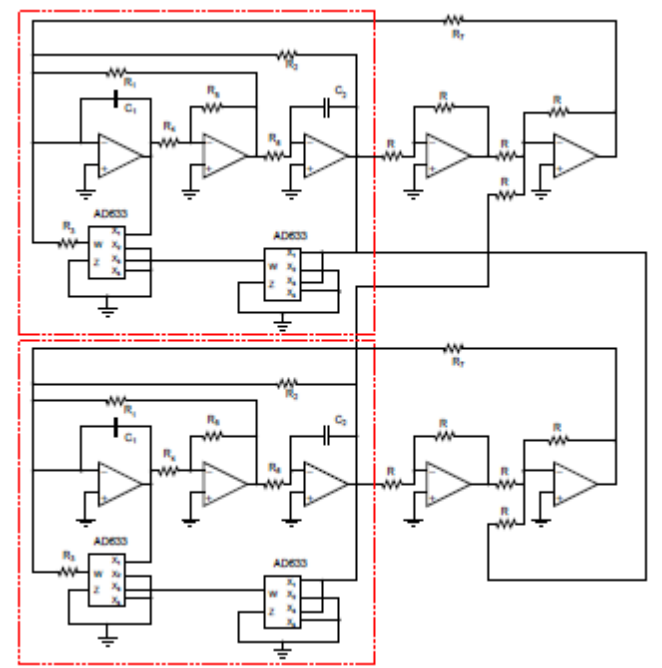

FIG. 8. Experimental circuit schematic of the two mutually coupled Van der Pol oscillator. 

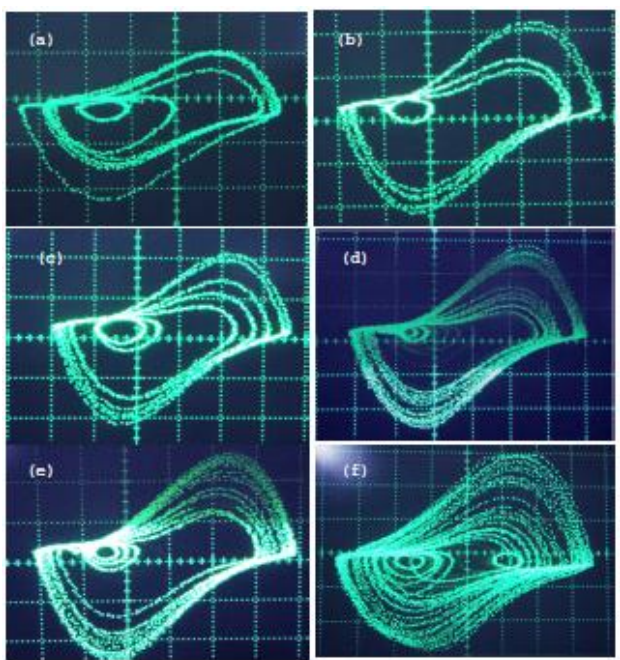

FIG. 9. Experimentally observed Phase portraits for different values of R7, Showing the period doubling cascades

write the following two equations,

$$
\begin{array}{r}
\ddot{y}-\frac{1}{C_{1} R_{1}}\left(1-y^{2}\right) \dot{y}+\frac{1}{C_{1} C_{2} R_{2} R_{7}} y-\frac{R_{11}}{R_{7} R_{10} R_{12} C_{1}}\left(y-y^{\prime}\right)=0 \\
\ddot{y}^{\prime}-\frac{1}{C_{1} R_{1}}\left(1-y^{2^{\prime}}\right) y^{\prime}+\frac{1}{C_{1} C_{2} R_{2} R_{7}} y^{\prime}-\frac{R_{11}}{R_{7} R_{10} R_{12} C_{1}}\left(y^{\prime}-y\right)=0
\end{array}
$$

In the above Eq. (6) equate to the equation number (2) the parameter are taken to adjust the resistor and capacitor values are $\omega 2$

$0=1$

R1R2R7C2 and $\mu 1,2=1$

$\mathrm{R} 1 \mathrm{C} 1 ! 0$ and the coupling co-efficient are $\mathrm{Q}=\mathrm{R} 11$

R7R10R12C1. But in our numerical study we have taken the parameter value's and $\mu 1=2.5, \mu 2=5.5$ and the coupling co-efficient is the control parameter. So, if our convenience we choose our experimental resistance and capacitance value's as follows

$\mathrm{R} 4, \mathrm{R} 5=10 \mathrm{~K}, \mathrm{R} 1, \mathrm{R} 6, \mathrm{R} 8-12=10 \mathrm{~K}, \mathrm{R} 3=1 \mathrm{~K}, \mathrm{C} 1, \mathrm{C} 2=10 \mathrm{nF}$ and the control parameter $\mathrm{R} 7$ varies from 0-150 $\mathrm{K}$ for the period doubling cascades. Such parameters let us show down experimental circuit to observe the phase portraits in HP Agilent 54601A 100MHz 4 Channel Oscilloscope. In the numerical analysis we have to determine the period doubling cascades in the very narrow regions. So, our experimental setup the resistance values are very short to observe phase portraits. The control parameter R7 is the value of $100 \mathrm{~K}$ to $101 \mathrm{~K}$ for the full length of period doubling cascades are occur.

\section{Conclusion}

To summarize, this paper describes the dynamics of two mutually coupled autonomous Van der pol oscillator realized by electronic circuit. We investigate the property of the anticipatory synchronization, existance of the chaotic attractor and the quasiperiodicity and it confirmed by bifurcation diagram, lyaponuv spectrum and power spectrum analysis,similarity functions, etc,. The existance of chaotic attractor also realized by numerical and realtime hardware experimental. In this study explores the mismatches of the relaxation oscillation of the two oscillator gives to choatic nature when they are mutually coupled. If the two oscillator having no mismatches then the two systems are synchronized this is also verified numerical as well as experimental. Extent the study to network of bidirectionally (mutually) coupled AVO's. We hope to devolope coupled AVO's in our nonlinear electronics laboratory suitably to perform the coupled dynamics of AVOs studies. 


\section{Acknowledgments}

S.S. acknowledge University Grants Commission (UGC) for the financial assistance through RFSMS scheme. K.T. acknowledges DST, Govt. of India for the financial support through the Grant no. SB/EMEQ- 077/2013.

\section{Reference}

[1]. I. Belykh, M. Hasler, M. Lauret and H. Nijmeijer, Syn- chronization and graph topology, Int. J. Bifurcation and Chaos, vol.15, no.11, pp.3423-3433, 2005.

[2]. R. Stoop and C. Wagner, Neocortex architecture opti- mizes computation, information transfer and synchronizability, at given total connection length,Int. J. Bifurcation and Chaos, vol.17, no.7, pp.2257-2279, 2007.

[3]. X.F. Wang and G. Chen, Synchronization in scale- free dynamical networks: robustness and fragility, IEEE Trans. Circuit and Systems-I, vol.49, no.1, pp.54-62, 2002.

[4]. Yoko Uwate, Yosifhimi Nishio and Ruedi Stoop, Synchro- nization in Three Coupled van der Pol Oscillators with Different Coupling Strength, NCSP10, Waikiki, Hawaii, March 3-5, 2010.

[5]. B. Nana and P. Woafo,[2006] Synchronization in a ring of four mutually coupled van der Pol oscillators: Theory and experiment, Phys. Rev. E 74, 046213, 2006.

[6]. A.P. Kuznetsov, E.P. Seleznev, N.V. Stankevich, Nonau- tonomous dynamics of coupled van der Pol oscillators in the regime of amplitude death, Commun Nonlinear Sci Numer Simulat 17, 3740^a“3746, 2012.

[7]. Pikovsky A, Rosenblum M, Kurths J, Synchronization: A Universal Concept in Nonlinear Science, England: Cambridge University Press, 2001.

[8]. Glass L, MacKey MC., From Clocks to Chaos, Princeton University Press; 1988.

[9]. P.Perlikowski,A. Stefanski,T. Kapitaniak, Discontinuous synchrony in an array of Van der Pol oscillators, International Journal of Non-Linear Mechanics 45, 895^a“901, 2010.

[10]. A.L. Barabasi, R. Albert, Emergence of scaling in ran- dom networks, Science 286, 509, 1999.

[11]. M. Rosenblum, A. Pikovsky, J. Kurths, Phase synchro- nization of chaotic oscillators, Phys. Rev. Lett. 76, 1804, 1996.

[12]. N.F. Rulkov, M.M. Sushchik, L.S. Tsimring, H.D.I. Abarbanel, Generalized synchronization of chaos in directionally coupled chaotic systems, Phys. Rev.E 51,980^a“999 ,1995.

[13]. D.J. Watts, S.H. Strogatz, Collective dynamics of small- world networks, Nature 393,440^a“4442, 1998.

[14]. T. Kapitaniak, M. Sekieta, M. Ogorzalek, Monotone synchronization of chaos, Int. J. Bifurcation Chaos 6, $211^{\wedge a}$ a“2 $215,1996$.

[15]. C.W. Wu, L. Chua, A unified framework for synchronization and control of dynamical systems, Int. J. Bifurcation Chaos 4,979^a“"998, 1996.

[16]. T. Yamada, H. Fujisaka, Stability theory of synchronized motion in coupled oscillator systems. II^a"the mapping approach, Prog. Theor. Phys. 70, (1983).

[17]. P. Perlikowski, S. Yanchuk, M. Wolfrum, A. Stefanski, P. Mosiolek and T. Kapitaniak, Routes to complex dynamics in a ring of unidirectionally coupled systems, Chaos 20, 013111, 2010.

[18]. B. van der Pol, Relaxation Oscillations, I. Phil. Mag., 2, pp. 978^a“992, 1926.

[19]. A. Mayer, On the theory of coupled vibrations of two self- excited generators, Proc. Gorky State Univ. 2, 1935.

[20]. V.I. Gaponov, Two coupled generators with soft self- excitation, Zh. Tech. Fiz. (J. Tech. Phys.) 6, 1936.

[21]. M. Rosenblum, A. Pikovsky, J. Kurths, C. Schaefer and P. Tass, Phase synchronization: from theory to data analysis, In: Handbook of Biological Physics, Elsevier Science, Series Editor A. J. Hoff, vol. 4: Neuro-informatics, Eds. F. Moss and S. Gielen, pp. 279-321, 2001.

[22]. G. Ambika, R. E. Amritkar,Anticipatory synchronization with variable time delay and reset, Phys. Rev.E 79, 056206, 2009.

[23]. H.U. Voss,Dynamic Long-Term Anticipation of ChaoticStates, Phys. Rev let, 87, 014102, 2001.

[24]. H.U. Voss, Coexistence of anticipated and layered chaotic synchronization in time-delay systems, Phys. Rev E, 72, 037203, 2005

[25]. K. Srinivasan, D. V. Senthilkumar, I. Raja Mohamed, K. Murali, M. Lakshmanan,Anticipating, complete and lag synchronizations in RC phase-shift network based coupled Chua aTMs circuits without delay, Chaos 22, 023124, 2012.

[26]. D. V. Senthilkumar and M. Lakshmanan,Delay time modulation induced oscillating synchronization and intermittent anticipatory/lag and complete synchronizations in time-delay nonlinear dynamical systems, Chaos 17, 013112, 2007.

[27]. M. Lakshmanan,D. V. Senthilkumar,Dynamics of Nonlinear Time-Delay Systems, Springer, 1st Edition., 2011. 